\title{
Improved Mode Selection in Coaxial Cavities for Sub-Terahertz Second-Harmonic Gyrotrons
}

\author{
Vitalii I. Shcherbinin, Member, IEEE, Yuliya K. Moskvitina, Konstantinos A. Avramidis, and \\ John Jelonnek, Senior Member, IEEE
}

\begin{abstract}
A coaxial metal rod with partial dielectric coating is considered as a means for efficient suppression of all volume competing modes in cavities for secondharmonic gyrotrons operated in whispering gallery modes. The rod radius is selected small enough to have only a slight effect on operating mode, which therefore remains insensitive to fabrication tolerances and a misalignment of the coaxial insert. By contrast, for the competing modes such a rod is shown to reduce the effective cavity length and thereby greatly increases the starting currents. Such a method of mode selection is demonstrated to be more versatile, as compared to that provided by a tapered coaxial conductor. The advantageous use of a dielectriccoated coaxial insert is illustrated by the example of a cavity for a $100-\mathrm{kW} 300-\mathrm{GHz}$ pulsed gyrotron operated in the second-harmonic mode.
\end{abstract}

Index Terms-Gyrotron, cyclotron harmonic, cavity, starting current, mode selection.

\section{INTRODUCTION}

$\mathrm{G}$ YROTRONS are powerful microwave oscillators, which have a wide field of applications. Among these applications is Collective Thomson Scattering (CTS) diagnostics of high-energy ions in fusion plasmas. In presentday fusion machines, such diagnostic method is employed with the help of gyrotrons operated at about $100 \mathrm{GHz}$ [1]-[3]. For these frequencies, however, the propagation of electromagnetic waves in the fusion plasma is often subjected to strong refraction and absorption, while the signal detection is impaired by the background noise from the electron cyclotron emission. To avoid these adverse effects in plasma diagnostics, pulsed gyrotrons, which produce more than $100 \mathrm{~kW}$ of output power in the frequency range $300-400 \mathrm{GHz}$, are needed [4]. Such high-frequency gyrotrons require

Manuscript received X.X.2020.

The work of V. I. Shcherbinin was supported by the Georg Forster Research Fellowship for Experienced Researchers from the Alexander von Humboldt Foundation.

V. I. Shcherbinin is with the Institute for Pulsed Power and Microwave Technology (IHM), Karlsruhe Institute of Technology (KIT), 76131 Karlsruhe, Germany, and also with the National Science Center "Kharkiv Institute of Physics and Technology", 61108 Kharkiv, Ukraine (e-mail:vshch@ukr.net).

Yu. K. Moskvitina is with the National Science Center "Kharkiv Institute of Physics and Technology", 61108 Kharkiv, Ukraine.

K. A. Avramidis and J. Jelonnek are with the Institute for Pulsed Power and Microwave Technology (IHM), Karlsruhe Institute of Technology (KIT), 76131 Karlsruhe, Germany. extremely large magnetic fields from superconducting magnets. Gyrotron operation at the second cyclotron harmonic makes it possible to relax this requirement. That is why highpower second-harmonic gyrotrons operated in sub-terahertz band are particularly attractive for use in CTS diagnostics of fusion plasmas. This fact has stimulated their development at the University of Fukui (FIR-UF), where second-harmonic operation of sub-terahertz gyrotrons has been demonstrated experimentally [5], [6]. However, in the experiments, the measured output power was found to be constrained by competition from first-harmonic (fundamental) modes. The upper limit of $83 \mathrm{~kW}$ was attained for the $390-\mathrm{GHz}$ gyrotron operated in the whispering gallery (WG) $\mathrm{TE}_{17,2}$ mode [6]. This achievement so far remains the power record of secondharmonic gyrotrons in sub-terahertz band. Thus mode competition makes the required power level of $100 \mathrm{~kW}$ inaccessible for sub-terahertz gyrotrons operated at the second cyclotron harmonic. For this reason, in CTS diagnostics of fusion plasmas, such gyrotrons are now abandoned in favor of first-harmonic counterparts. For the oscillation frequency of about $300 \mathrm{GHz}$, first-harmonic gyrotrons equipped with $12 \mathrm{~T}$ superconducting magnets are now designed at FIR-UF and can operate in the WG $\mathrm{TE}_{14,2}$ and $\mathrm{TE}_{22,2}$ modes with an output power in excess of 200 and $300 \mathrm{~kW}$ [7], [8], respectively.

Whispering gallery modes are known to feature field localization near the cavity walls. Therefore, compared to volume modes, these modes are less sensitive to conductors placed near the cavity axis. This fact can be used for mode selection (see [9] and the references therein). For secondharmonic gyrotrons such mode selection can be realized by tapered coaxial rods with longitudinal impedance corrugations [10], resistive rods [10], [11], and stepped inner conductors [12]. Thus the use of a coaxial insert may remove the constraint on power increase for second-harmonic gyrotrons operated at WG modes and thereby can provide the missing link for their application in CTS diagnostics of fusion plasmas. It should be noted, however, that none of the available methods of mode selection in coaxial cavities, with the exception of that provided by resistive rods, may ensure efficient suppression of all volume competing modes in a second-harmonic gyrotron, i.e. modes having smaller caustic radii than that of an operating mode. In short, this is because the eigenvalues of competing modes of a coaxial gyrotron cavity are different functions of the outer-to-inner radius ratio. Thus, even with these methods, the design of coaxial-cavity 
second-harmonic gyrotrons for CTS can be quite challenging. To facilitate such a design, in this paper we propose a novel concept based on equipping the gyrotron cavity with a uniform inner rod, which is partially coated by a thin dielectric layer. The beneficial property of such a rod is its ability to provide essentially the same eigenvalue variation along the cavity axis for all volume modes. Owing to this property, it may be particularly useful for improvement of the mode selection in gyrotron cavities of second-harmonic gyrotrons.

The paper is organized as follows. In Section II, the use of a tapered metal coaxial rod is investigated with the aim to suppress the competing modes in a cavity of a secondharmonic gyrotron. Some advantages and disadvantages of mode selection in coaxial cavities are shown and discussed. In Section III, as a step towards further improvement of mode selection in gyrotron cavities, the use of a uniform coaxial rod with partial dielectric coating is proposed and investigated. Such a use of a dielectric-coated insert is illustrated by an example of a cavity for a $100-\mathrm{kW} 300-\mathrm{GHz}$ gyrotron operated in a second-harmonic WG mode. In the last section, the results of the investigation are summarized and discussed.

\section{CONVENTIONAL CYLINDRICAL AND COAXIAL CAVITIES}

The aim of our theoretical investigation is to design a cavity, which ensures single-mode operation for a pulsed second-harmonic gyrotron with an oscillation frequency of about $300 \mathrm{GHz}$ and a peak output power in excess of $100 \mathrm{~kW}$. As far as mode competition is concerned, it is safe to say that single-mode gyrotron operation is feasible if the starting currents of all competing modes exceed the operating beam current in a relatively wide range of the beam parameters.

Assume that a $300-\mathrm{GHz}$ gyrotron is originally equipped with cylindrical cavity $\mathbf{A}$ and has typical beam parameters [7], [8]: the beam voltage $V_{b}=60 \mathrm{kV}$, the beam current $I_{b}=10$ A, and the pitch factor $\alpha=1.2$. In deciding on an operating mode, we are guided by the simple rule, which implies that the operating mode should not be subjected to strong competition from the $\mathrm{TE}_{m, n}$ modes having comparable or larger caustic radii $R_{m n}=R|m| / \mu_{m n}$, where $R$ is the cavity radius, $\mu_{m n}$ satisfies the equation $J_{m}^{\prime}\left(\mu_{m n}\right)=0$, and $J_{m}(\cdot)$ is the $m$-order Bessel function. On this basis, we select the $\mathrm{TE}_{13,2}$ mode and $R=0.32 \mathrm{~cm}$. The other parameters of conventional threesection cavity $\mathbf{A}$ are as follows: the input and output taper angles are both equal to $3^{\circ}$, the lengths of the input, central (main) and output sections are $L_{1}=1 \mathrm{~cm}, L_{2}=1.3 \mathrm{~cm}$, $L_{3}=1 \mathrm{~cm}$, respectively. The cavity is assumed to be made of copper with a conductivity $2.9 \times 10^{7} \mathrm{~S} / \mathrm{m}$ [8]. The beam radius is set to $0.2 \mathrm{~cm}$ in order to maximize the beam coupling with the operating $\mathrm{TE}_{13,2}$ mode.

In simulations, we use self-consistent single-mode code KIPT [13], [14] benchmarked recently (see [12]) against single-mode results of the European code-package EURIDICE [15], [16]. The code KIPT relies on well-known simplified gyrotron equations [17], [18] extended to account for selfconsistent effect of ohmic losses in a metal gyrotron cavity on a beam-wave interaction efficiency (see equations (3) in [13]). In this code, the starting current of a cavity mode is approximated by the beam current, which satisfies the selfconsistent gyrotron equations for very small mode amplitude. In the following, it is assumed that the velocity spread of the beam electrons has a slight effect on the beam-wave interaction in a gyrotron cavity. This assumption is known to be good for low-order axial modes [18].

For the cylindrical cavity $\mathbf{A}$ the starting currents of the competing modes versus the operating magnetic field $B_{0}$ are shown in Fig. 1. As this figure indicates, the mode spectrum is relatively dense for the $300-\mathrm{GHz}$ second-harmonic gyrotron operated in the $\mathrm{TE}_{13,2}$ mode. It is seen from Fig. $1 \mathrm{~b}$ that none of the second-harmonic modes can be in fact considered as a serious competitor of the operating $\mathrm{TE}_{13,2}$ mode, which is the only second-harmonic mode capable of oscillating at the operating beam current $I_{b}=10 \mathrm{~A}$ in the vicinity of $B_{0}=5.82 \mathrm{~T}$. Competition from second-harmonic modes is possible beyond this vicinity and therefore can make itself evident with frequency tuning. It should be stressed that the competing $\mathrm{WG} \mathrm{TE}_{8,1}, \mathrm{TE}_{12,2}$ and $\mathrm{TE}_{14,2}$ modes are beyond the operating region of the $300-\mathrm{GHz}$ second-harmonic gyrotron (see Fig. 1), as desired.

The most dangerous competing modes are the first-
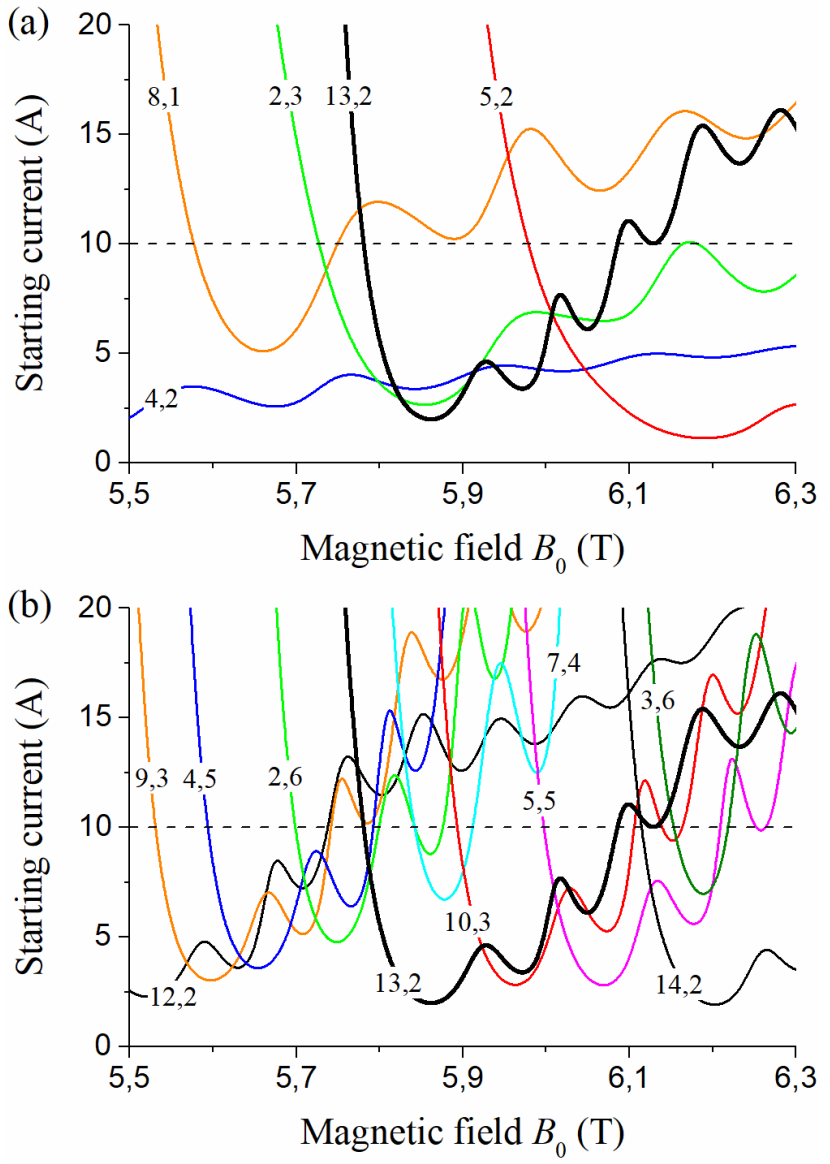

Fig. 1. Starting currents of the operating $T E_{13,2}$ mode and (a) first- and (b) second-harmonic competing modes of the $300-\mathrm{GHz}$ secondharmonic gyrotron with cylindrical cavity $\mathbf{A}$. 
harmonic volume modes, as indicated in Fig. 1a. Special attention must be paid to the $\mathrm{TE}_{2,3}$ mode. The starting current of this mode is close to that of the operating mode. Moreover, in the operating range of the $300-\mathrm{GHz}$ second-harmonic gyrotron, the $\mathrm{TE}_{2,3}$ mode, as opposed to the first-harmonic $\mathrm{TE}_{4,2}$ mode, can oscillate at low-order axial resonances. Therefore, it is not expected that this mode, once excited, will suffer from the velocity spread of the beam electrons. This suggests a strong competition between the operating whispering gallery $\mathrm{TE}_{13,2}$ mode and the competing volume $\mathrm{TE}_{2,3}$ mode.

The volume competing modes can be disturbed by a coaxial conductor inserted into the gyrotron cavity. The effect of a coaxial copper rod of radius $R_{i}$ on the cutoff frequencies (eigenvalues $\chi_{m n}$ ) of the waveguide with radius $R=0.32 \mathrm{~cm}$ is shown in Fig. 2. It is seen that the operating $\mathrm{TE}_{13,2}$ mode is almost insensitive to the inner conductor, provided that the outer-to-inner radius ratio $C=R / R_{i}$ is greater than 2.2. In this case, the fabrication imperfections of the coaxial insert have little or no effect on the operating mode. For this reason we adopt the condition $C=2.2$ as an upper limit on the insert radius $R_{i}$ suitable for mode selection in the coaxial-cavity gyrotron operated in the second-harmonic $\mathrm{TE}_{13,2}$ mode.

There are three major factors, which may assist in such mode selection. First, the coaxial insert affects the eigenvalue spectrum and therefore can be applied to shift the frequencies of dangerous competing modes beyond the operating region of the gyrotron. This can be done, for instance, for the most dangerous competing $\mathrm{TE}_{2,3}$ mode of the $300-\mathrm{GHz}$ secondharmonic gyrotron, provided that $C<2.6$ (see Fig. 2a). On the other hand, the coaxial inner conductor can reduce the frequency separation between the operating mode and some competing modes, thereby making mode selection in the gyrotron cavity more complicated. Moreover, the coaxial insert may give rise to new competitors in the vicinity of the operating frequency. As is seen from Fig. 2, for the $300-\mathrm{GHz}$ second-harmonic gyrotron such competitors are the firstharmonic $\mathrm{TE}_{1,3}$ mode and the second-harmonic $\mathrm{TE}_{1,5}$ and $\mathrm{TE}_{2,5}$ modes. Despite these shortcomings, the use of a coaxial insert may enable one to rarify the spectrum of the cavity modes in some cases [9].

Second, the $\mathrm{TE}_{\mathrm{m}, \mathrm{n}}$ modes of a coaxial cavity have different dependence of the eigenvalue $\chi_{m n}$ on the radius ratio $C=R / R_{i}$. Therefore, the diffraction losses of these modes can be differently affected by a variation of $C$ along the cavity axis (z-axis) and are identical to those in cylindrical cavities with equivalent radii $R_{e q}=R \mu_{m n} / \chi_{m n}(C)$. Assume that $C$ and $\chi_{m n}$ vary continuously with $z$. If $\chi_{m n}$ is a decreasing function of $z$, then the equivalent radius $R_{e q}(z)$ corresponds to an up-tapered gyrotron cavity. In this case, the diffraction losses of the $\mathrm{TE}_{\mathrm{m}, \mathrm{n}}$ mode increase with increasing gradient of $\chi_{m n}(C)$. In the opposite case, $R_{e q}(z)$ presents the radius-profile of a down-tapered cylindrical cavity, which

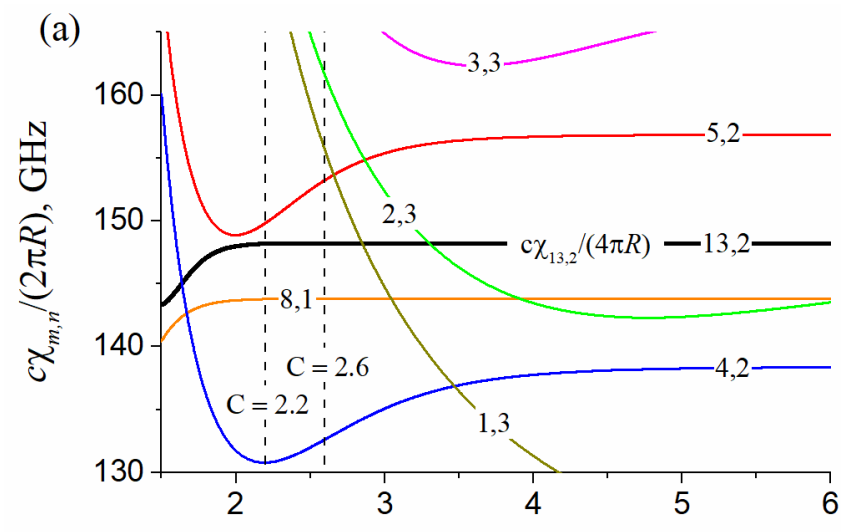

Outer-to-inner radius ratio $C$

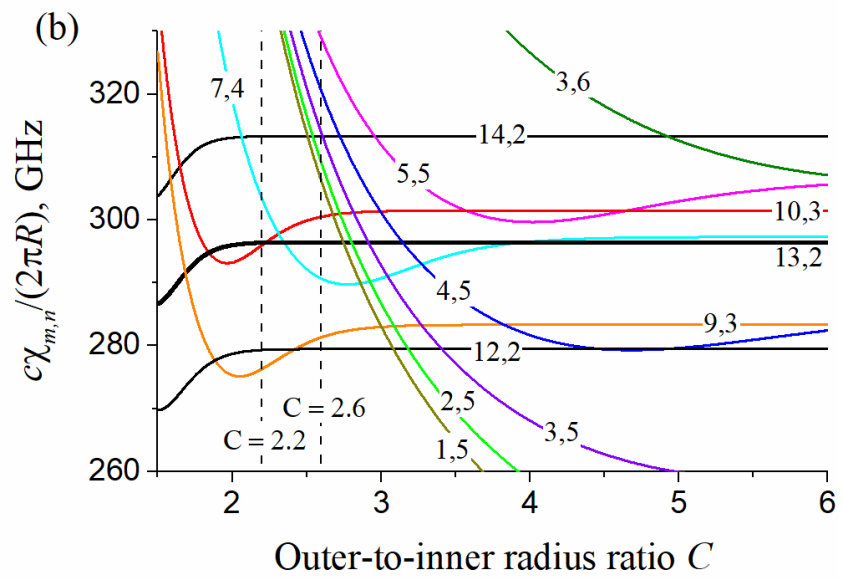

Fig. 2. Cutoff frequencies versus radius ratio $C=R / R_{i}$ for the (a) firstand (b) second-harmonic modes of a coaxial waveguide with outer radius $R=0.32 \mathrm{~cm}$.

features lower diffraction losses in comparison with those of a uniform cavity. From the above discussion (see also [19]), it seems reasonable to equip the cavity $\mathbf{A}$ with up-tapered inner conductor, which is characterized by the variation of $C$ from $2.6\left(R_{i}=0.123 \mathrm{~cm}\right)$ to $2.2\left(R_{i}=0.145 \mathrm{~cm}\right)$ along the central cavity section (see Fig. 2). The resulting coaxial cavity will be called cavity B. It is expected that, in this cavity, the diffraction losses and starting currents of dangerous competing modes (e.g. $\mathrm{TE}_{5,2}$ and $\mathrm{TE}_{10,3}$ modes) will increase as compared to those in the cylindrical cavity $\mathbf{A}$.

Third, the coaxial inner conductor contributes to the ohmic losses in the gyrotron cavity. This contribution increases with $R_{i}$ and is larger for modes with lower caustic radii. Therefore, in coaxial cavities, the suppression of competing modes by ohmic losses is possible. To be efficient, such mode suppression, however, requires conductivity of the inner conductor to be sufficiently low [10], [11], [19]. In this case, special care must be taken in selecting proper material and adequate cooling of the coaxial insert in order to mitigate the risk of high thermal loading (especially when designing highpower gyrotrons). Therefore, in the following, for cavity $\mathbf{B}$ we consider a good-conducting insert, which is made of copper with assumed conductivity of $2.9 \times 10^{7} \mathrm{~S} / \mathrm{m}$. In this case, cavity 
ohmic losses do not play a key role in mode selection, especially for high-order axial modes characterized by high diffraction losses [14].

Fig. 3 shows starting currents of the operating and competing modes of the $300-\mathrm{GHz}$ second-harmonic gyrotron with coaxial cavity B. In this cavity, all second-harmonic volume modes are selectively suppressed by the inner conductor (Fig. 3b). In order to explain this fact let us first look at the $\mathrm{TE}_{10,3}$ mode of the coaxial cavity $\mathbf{B}$. For this mode the eigenvalue $\chi_{m n}$ decreases along the cavity axis (Fig. 2b) and thus $R_{e q}(z)$ describes an up-tapered equivalent cylindrical cavity (Fig. 4). That is why coaxial insert leads to a rise in the diffraction losses and starting current of the $\mathrm{TE}_{10,3}$ mode. Another situation takes place with the $\mathrm{TE}_{7,4}$ mode of the coaxial cavity B. For this mode the eigenvalue $\chi_{m n}$ increases with $z$ and radius-profile $R_{e q}(z)$ corresponds to a downtapered equivalent cylindrical cavity (Fig. 4). In this cavity, the $\mathrm{TE}_{7,4}$ mode exhibits low diffraction losses. Generally, the lower are the diffraction losses, the lower is the starting current. At the same time, cavity down-tapering reduces the effective cavity length and thereby tends to increase the starting current. This effect becomes dominant in downtapered cavities characterized by low power of outgoing radiation. Such is the case for low-order axial resonances of
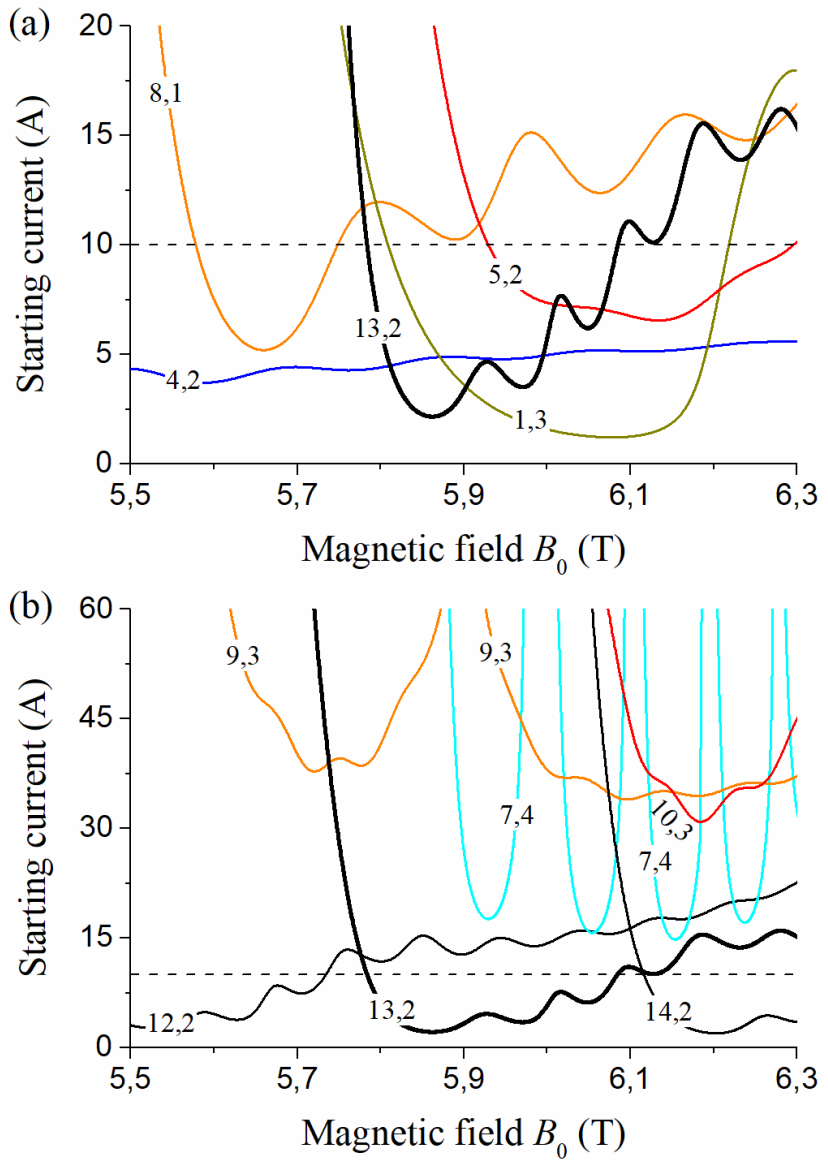

Fig. 3. The same as in Fig. 1, but for coaxial cavity B with uptapered inner conductor.

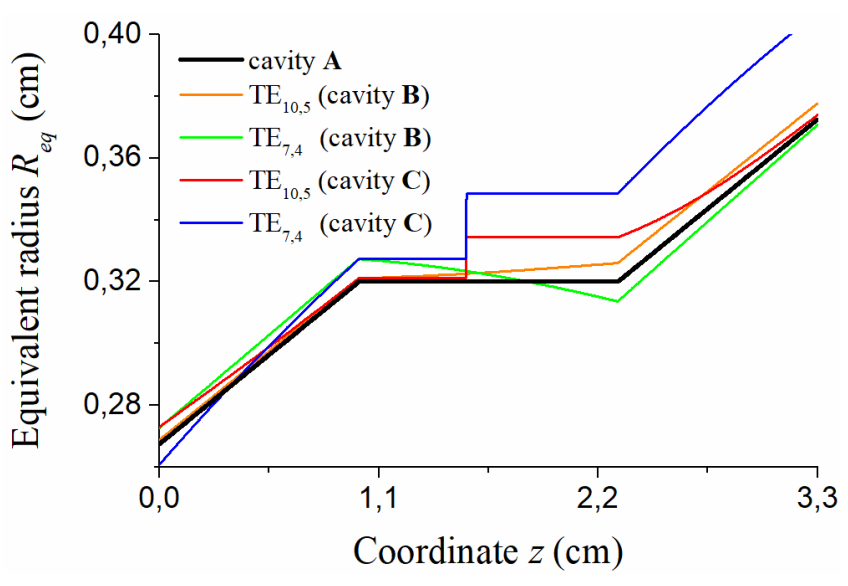

Fig. 4. Equivalent radii $R_{e q}(z)=R(z) \mu_{m n} / \chi_{m n}(z)$ for the $\mathrm{TE}_{7,4}$ and $\mathrm{TE}_{10,5}$ modes of coaxial cavities B and C $\left(z_{d}=1.54 \mathrm{~cm}\right)$. In cylindrical cavity A, the condition $R_{e q}(z)=R(z)$ holds true.

the $\mathrm{TE}_{7,4}$ mode. For these resonances, the mode attenuation is mainly determined by the ohmic losses inherent in the metal cavity surface.

The above-described influence of the tapered coaxial insert on the starting current refers to all first- and second-harmonic competing modes of the cavity $\mathbf{B}$ and generally improves mode selection in the $300-\mathrm{GHz}$ second-harmonic gyrotron. Despite this improvement, however, such mode selection is not always adequate to completely avoid mode competition. For the coaxial cavity $\mathbf{B}$, examples are provided by the firstharmonic competing $\mathrm{TE}_{1,3}$ and $\mathrm{TE}_{4,2}$ modes. As is seen from Fig. 3a, these modes show themselves as dangerous competitors, which are capable of rendering operation of the 300-GHz second-harmonic gyrotron impossible.

Thus, stable single-mode operation of the $100-\mathrm{kW} 300-\mathrm{GHz}$ second-harmonic gyrotron calls for a better choice of the operating mode or the use of an alternative structure of the gyrotron cavity. One of such alternatives is a coaxial cavity with corrugated insert incorporating subwavelength-sized longitudinal corrugations. Such corrugations are known as impedance corrugations and can be described by the surface impedance model (SIM) [10], [20]. It is common knowledge that, in a coaxial gyrotron cavity, a down-tapered rod equipped with impedance corrugations provides monotonic eigenvalue curves with negative slope for all volume first-harmonic modes having the wavelength $\lambda$ within the range $h / \lambda=0.2 \div 0.3$, where $h$ is the corrugation depth [10], [20], and thus it leads to suppression of the first-harmonic competitors [10]. However, in this case, second-harmonic modes, which are subject to the condition $h / \lambda=0.4 \div 0.6$, feature non-monotonic eigenvalue curves [10], [20]. Therefore, under this condition, longitudinally corrugated insert may facilitate oscillation of several second-harmonic competing modes, thereby making them more dangerous competitors. Moreover, the subwavelength condition $p<<\lambda$ of SIM validity is difficult to satisfy for corrugations with 
$h / \lambda=0.4 \div 0.6$, provided that the depth $h$ does not exceed the corrugation period $p$. Otherwise, to take advantage of impedance corrugations, one would have to use a tapered inner conductor with deep narrow slits, which are difficult to fabricate. With the above-described difficulties in mind, in the following we restrict our consideration to coaxial cavities with smooth (non-corrugated) inserts.

For mode selection in such cavities it is sometimes advantageous to use coaxial rods with stepped variation of the inner radius [12]. The reason lies in the flexibility of such a design solution, which can be applied to discriminate against competing modes having non-monotonic eigenvalue curves in the required range of $C$ (see, for example, the eigenvalue of the $\mathrm{TE}_{3,5}$ mode in Fig. 5 of [12]). However, even this design does not suffice to suppress all volume competing modes (see, for example, the $\mathrm{TE}_{4,2}$ mode of [12]). In the next section, we propose an alternative structure of a coaxial cavity, which is free from this shortcoming.

\section{COAXIAL CAVITY WITH DIELECTRIC-COATED INSERT}

It is proposed to equip cavity $\mathbf{A}$ with a uniform copper rod partially coated by dielectric. The dielectric coating is in the form of a low-loss ( $\tan \delta<0.0005)$ PTFE tube with the relative permittivity $\varepsilon=2$. Such tubes of various dimensions are now commercially available and can operate at temperatures up to about $260^{\circ} \mathrm{C}$ (see, for example, [21]). The PTFE coating is considered to have a thickness $d$ of $0.02 \mathrm{~cm}$.

Fig. 5 shows the cutoff frequencies of a coaxial waveguide with outer radius $R=0.32 \mathrm{~cm}$ and radius ratio $C$ for $d=0$ (see also Fig. 2) and $d=0.02 \mathrm{~cm}$. It can be seen that the dielectric coating of the inner rod reduces the cutoff frequencies. Therefore, the coating must be selected relatively thin to avoid occurrence of additional higher-order modes in the vicinity of the operating frequency. Since dielectric coating reduces the eigenvalue $\chi_{m n}$, the equivalent radius $R_{e q}$ increases with $d$ for any $m$ and $n$. For this reason, a partial dielectric coating of the inner conductor results in essentially the same form of radius profile $R_{e q}(z)$ for all volume modes of a coaxial gyrotron cavity. This property is described in more detail below and distinguishes a dielectric-coated insert from an uncoated tapered rod, which can lead to either increasing or decreasing function $R_{e q}(z)$, depending on azimuthal and radial mode indices (see results for cavity $\mathbf{B}$ in Fig. 4).

To achieve improved mode selection, we equip the cylindrical cavity $\mathbf{A}$ with a uniform copper rod of radius $R_{i}=0.123 \mathrm{~cm}(C=2.6)$. The rod consists of two parts. The input part is uncoated $(d=0)$ and extends from the cavity input to the coordinate $z_{d}$. The remainder of the rod is coated by the PTFE layer with the thickness $d=0.02 \mathrm{~cm}$ and is responsible for increased values of $R_{e q}$ at $z>z_{d}$ for all volume modes. The resulting coaxial cavity will be called cavity $\mathbf{C}$. For any volume $\mathrm{TE}_{m, n}$ mode of the cavity $\mathbf{C}$, the
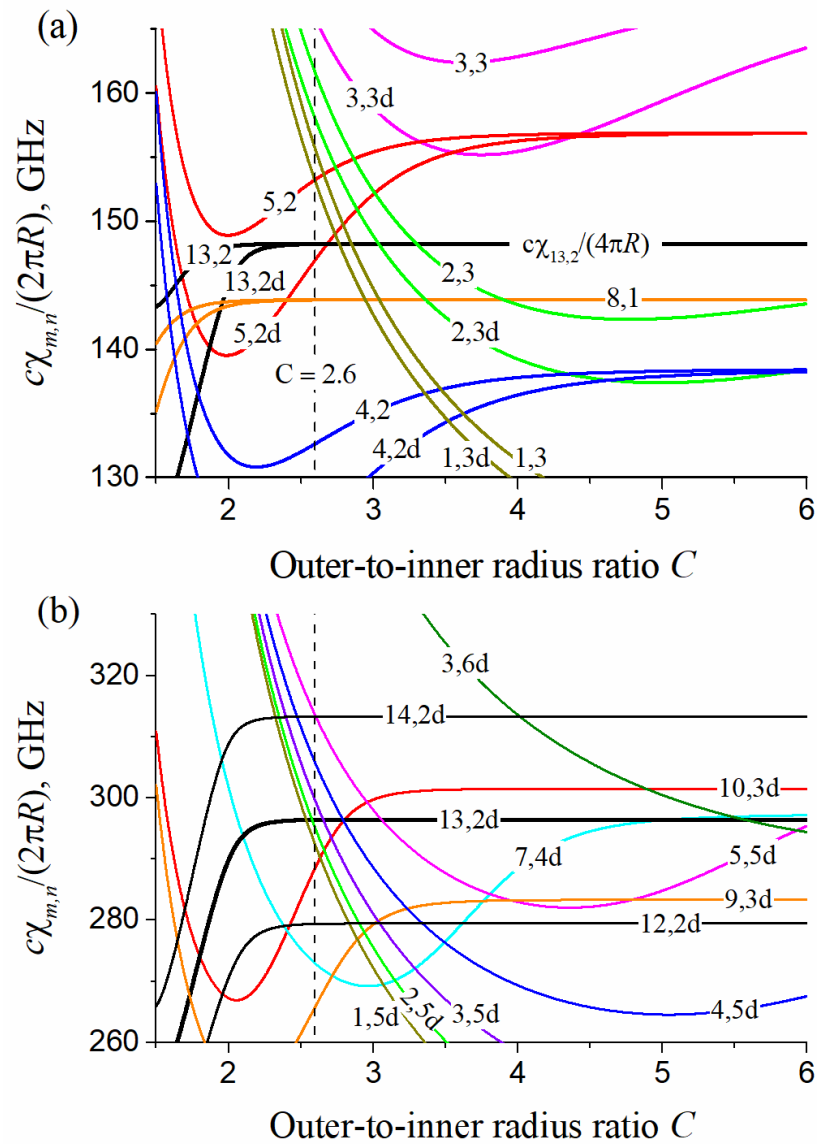

Fig. 5. Cutoff frequencies versus radius ratio $C=R / R_{i}$ for the (a) firstand (b) second-harmonic modes of a coaxial waveguide with outer radius $R=0.32 \mathrm{~cm}$ and either uncoated ( $d=0$ ) or dielectric-coated $(\varepsilon=2, d=0.02 \mathrm{~cm}$ ) copper rod. In the case of $d=0.02 \mathrm{~cm}$, TE modes are marked by the letter "d".

radius profile $R_{e q}(z)=R(z) \mu_{m n} / \chi_{m n}(z)$ has a jump discontinuity at $z_{d}$ and resembles the profile of a complex gyrotron cavity (see results for cavity $\mathbf{C}$ in Fig. 4). It is wellknown that in a complex cavity, any competing mode is reflected from the wall discontinuity [22]-[24]. This gives rise to two modes, which are mostly localized inside the left and right parts of the cavity volume. In cavity $\mathbf{C}$, such modes are excited by the electron beam near the cutoff frequencies shown in Figs. 2 and 5 for $d=0$ and $d=0.02 \mathrm{~cm}$, respectively. When compared to the modes of the original cavity $\mathbf{A}$, these modes have smaller regions of the field localization and therefore exhibit higher starting currents. In the following, we set the coordinate $z_{d}$ equal to $1.54 \mathrm{~cm}$ in order to suppress all volume competing modes localized in both the left and right parts of the cavity $\mathbf{C}$.

To investigate the operation of the $300-\mathrm{GHz}$ secondharmonic gyrotron with the cavity $\mathbf{C}$, the code KIPT has been extended to take into account the effect of dielectric coating on mode eigenvalues and mode coupling with the electron beam. In the vicinity of the cutoff frequencies, the cavity modes of interest are (quasi-) TE modes [25]. Their 

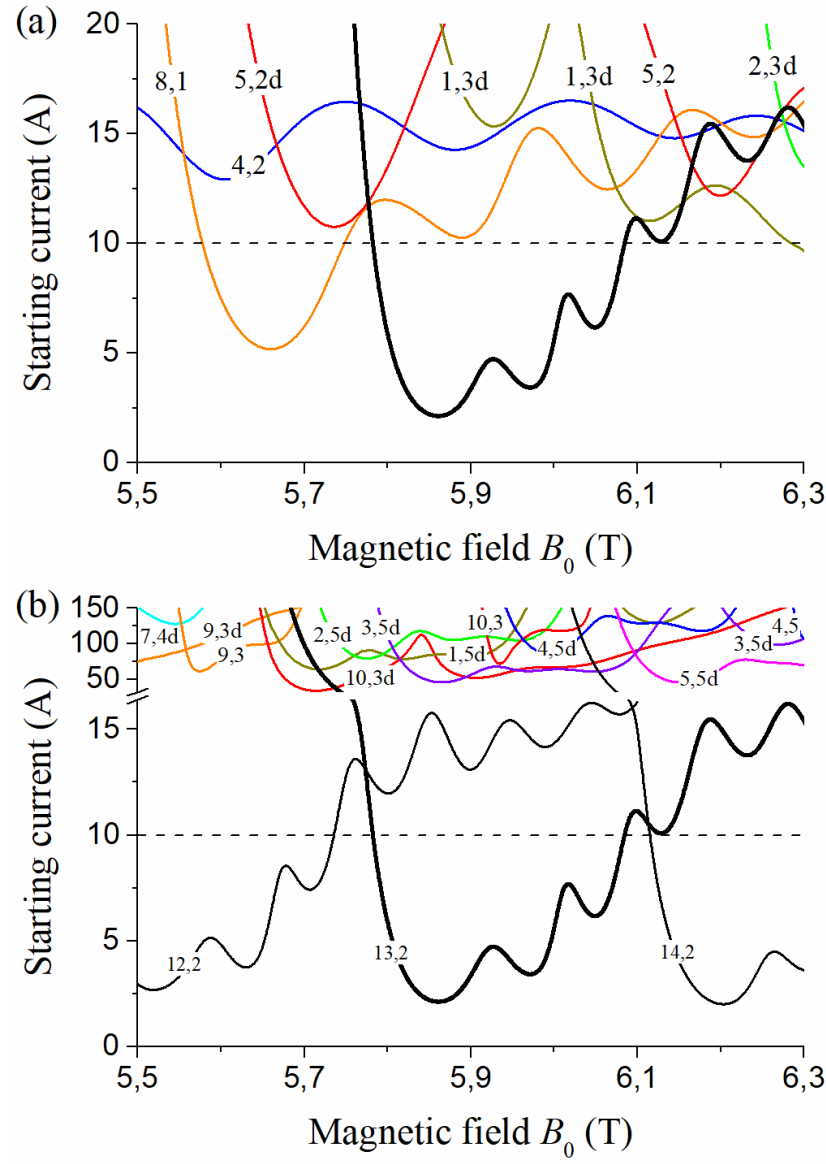

Fig. 6. The same as in Fig. 1, but for coaxial cavity $\mathbf{C}$ with dielectriccoated inner conductor.

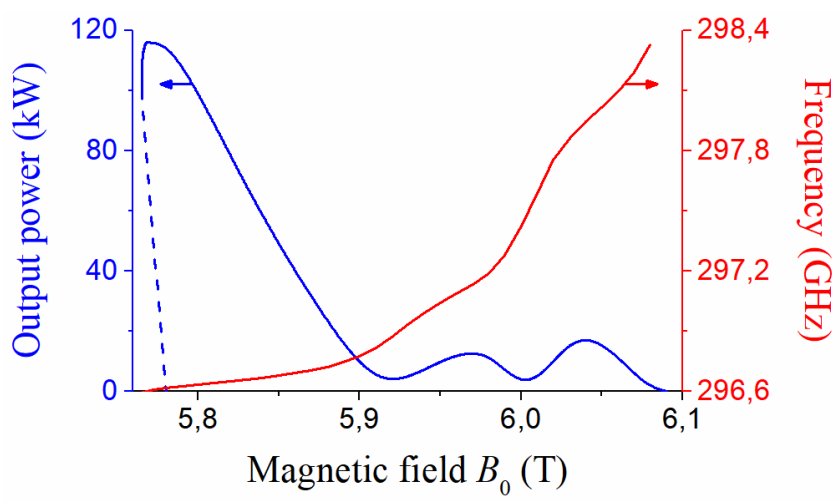

Fig. 7. Output power and frequency of the $300-\mathrm{GHz}$ second-harmonic gyrotron with coaxial cavity $\mathbf{C}$.

eigenvalues and beam-wave coupling coefficients can be found from rigorous consideration [26], [27]. At the same time, for a small dielectric thickness $d$, when the field inside a dielectric layer has a small contribution to the power flow along the cavity, an approximation can be used. In this approximation, the dielectric-coated metal surface can be treated as an impedance surface [27]. In such case, the theoretical description of beam-wave interaction in a dielectric-loaded cavity takes the same form as for a cavity with impedance surface [13], e.g. imperfectly conducting surface [13] or corrugated surface [28], [29].

Fig. 6 shows starting currents of the operating and competing modes of the $300-\mathrm{GHz}$ second-harmonic gyrotron with coaxial cavity $\mathbf{C}$. It is seen that, in this cavity, all volume competing modes are selectively suppressed by the dielectriccoated inner conductor. As a result, one can expect stable single-mode operation of the $300-\mathrm{GHz}$ second-harmonic gyrotron in a relatively wide range of magnetic fields from 5.78 to $6.08 \mathrm{~T}$. In this range, the operating second-harmonic $\mathrm{TE}_{13,2}$ mode is the only oscillating mode. Therefore, there are no obstacles for this mode to oscillate stably. For $B_{0}=5.57 \mathrm{~T}$ the operating mode attains a peak output power of $116 \mathrm{~kW}$ (Fig. 7), which meets the design goal. This demonstrates that the improved mode selection in a coaxial cavity with dielectric-coated insert allows sub-terahertz second-harmonic gyrotrons to achieve the high performance required for CTS diagnostics of fusion plasmas.

\section{CONCLUSION}

In the sub-terahertz to terahertz frequency band, the development of electromagnetic applications continuously generates a need for high-power oscillators operated at higher and higher frequencies. Second-harmonic gyrotrons with medium-field magnets are naturally suited to these applications. Their wide use in practice, however, is mainly hindered by the competition from the first-harmonic modes. For this reason, the feasibility of second-harmonic gyrotrons often requires highly efficient methods of mode selection.

It has been shown that the required mode selection in a gyrotron cavity can be achieved with uniform copper rod partially coated by dielectric. The dielectric coating of the coaxial insert has no effect on the operating mode, but introduces reflection of the competing modes. As a result, these competing modes exhibit reduced length of the field localization and increased diffraction losses in the gyrotron cavity. It has been demonstrated that such effect of a dielectric-coated coaxial insert tends to increase the starting currents of all competing modes having smaller caustic radii than that of the operating mode. This property distinguishes the proposed approach from the well-known method of mode selection by tapered coaxial rods, which can have a dissimilar effect on the competing modes and therefore can be sometimes inadequate to avoid mode competition in secondharmonic gyrotrons. The dielectric coating has been considered by the example of commercially available thinwall PTFE tube. Such a coating is used as an illustration and could be an inappropriate choice for gyrotron, in view of the vacuum, thermal, mechanical or charging properties of PTFE. Other dielectric materials (e.g. quartz or diamond), which find use in terahertz dielectric-loaded RF structures of highgradient wakefield accelerators [30]-[32], can be more suitable for use in cavities of sub-terahertz gyrotrons. The technical feasibility of such gyrotron cavities is beyond the scope of this paper and calls for further investigation. 


\section{REFERENCES}

[1] H. Bindslev, S. K. Nielsen, L. Porte, J. A. Hoekzema, S. B. Korsholm, F. Meo, P. K. Michelsen, S. Michelsen, J. W. Oosterbeek, E. L. Tsakadze, E. Westerhof, and P. Woskov, "Fast-ion dynamics in the TEXTOR Tokamak measured by collective Thomson scattering," Phys. Rev. Lett. vol. 97, no. 20, p. 205005, Nov. 2006, doi: 10.1103/PhysRevLett.97.205005.

[2] M. Nishiura, S. Kubo, K. Tanaka, R. Seki, S. Ogasawara, T. Shimozuma, K. Okada, S. Kobayashi, T. Mutoh, K. Kawahata, T. Watari, T. Saito, Y. Tatematsu, S.B. Korsholm, and M. Salewski, "Spectrum response and analysis of $77 \mathrm{GHz}$ band collective Thomson scattering diagnostic for bulk and fast ions in LHD plasmas," Nucl. Fusion, vol. 54, no. 2, p. 023006, Jan. 2014, doi: 10.1088/00295515/54/2/023006.

[3] J. Rasmussen, S. K. Nielsen, M. Stejner, J. Galdon-Quiroga, M. GarciaMunoz, B. Geiger, A.S. Jacobsen, F. Jaulmes, S.B. Korsholm, N Lazanyi, F. Leipold, F. Ryter, M. Salewski, M. Schubert, J. Stober, and D. Wagner, "Collective Thomson scattering measurements of fast-ion transport due to sawtooth crashes in ASDEX Upgrade," Nucl. Fusion vol. 56, no. 11, p. 112014, Jul. 2016, doi: 10.1088/0029$\underline{5515 / 56 / 11 / 112014}$

[4] T. Notake, T. Saito, Y. Tatematsu, S. Kubo, T. Shimozuma, K. Tanaka M. Nishiura, A. Fujii, L. Agusu, I. Ogawa, and T. Idehara, "Subterahertz gyrotron developments for collective Thomson scattering in LHD, " Rev Sci. Instrum., vol. 79, no. 10, p. 10E732, Oct. 2008, doi: 10.1063/1.2966372.

[5] T. Notake, T. Saito, Y. Tatematsu, A. Fujii, S. Ogasawara, L. Agusu, I. Ogawa, T. Idehara, and V. N. Manuilov, "Development of a novel high power sub-THz second harmonic gyrotron," Phys. Rev. Lett., vol. 103, no. 22, p. 225002, Nov. 2009, doi: 10.1103/PhysRevLett.103.225002.

[6] T. Saito, N. Yamada, S. Ikeuti, S. Ogasawara, Y. Tatematsu, R. Ikeda, I Ogawa, T. Idehara, V. N. Manuilov, T. Shimozuma, S. Kubo, M Nishiura, K. Tanaka, and K. Kawahata, "Generation of high power subterahertz radiation from a gyrotron with second harmonic oscillation," Phys. Plasmas, vol. 19, no. 6, p. 063106, June 2012, doi 10.1063/1.4729316.

[7] Y. Yamaguchi, T. Saito, Y. Tatematsu, S. Ikeuchi, V. N. Manuilov, J. Kasa, M. Kotera, T. Idehara, S. Kubo, T. Shimozuma, K. Tanaka, and M. Nishiura, "High-power pulsed gyrotron for $300 \mathrm{GHz}-$ band collective Thomson scattering diagnostics in the Large Helical Device," Nucl. Fusion, vol. 55, no. 1, p. 013002, Jan. 2015, doi: 10.1088/00295515/55/1/013002

[8] T. Saito, S. Tanaka, R. Shinbayashi, Y. Tatematsu, Y. Yamaguchi, M. Fukunari, S. Kubo, T. Shimozuma, K. Tanaka, and M. Nishiura, "Oscillation characteristics of a high power $300 \mathrm{GHz}$ band pulsed gyrotron for use in collective Thomson scattering diagnostics, " Plasma Fusion Res., vol. 14, p. 1406104, Jul. 2019, doi: 10.1585/pfr.14.1406104.

[9] O. Dumbrajs and G. S. Nusinovich, "Coaxial gyrotrons: Past, present, and future (review)," IEEE Trans. Plasma Sci., vol. 32, no. 3, pp. 934 946, Jun. 2004, doi: 10.1109/TPS.2004.829976.

[10] K. A. Avramides, C. T. Iatrou, and J. L. Vomvoridis, "Design considerations for powerful continuous-wave second-cyclotronharmonic coaxial-cavity gyrotrons," IEEE Trans. Plasma Sci., vol. 32, no. 3, pp. 917-928, Jun. 2004, doi: 10.1109/TPS.2004.828781.

[11] K. A. Avramides, J. L. Vomvoridis, and C. T. Iatrou, "Coaxial gyrotron cavities with resistive corrugated insert for powerful second-harmonic operation," AIP Conference Proceedings, vol. 807, pp. 264-270, Jan. 2006. 10.1063/1.2158787.

[12] V. I. Shcherbinin, V. I. Tkachenko, K. A. Avramidis, and J. Jelonnek, "Coaxial cavity with stepped inner conductor for a sub-terahertz secondharmonic gyrotron with broadband continuous frequency tuning," IEEE Trans. Electron Devices, vol. 66, no. 12, pp. 5313-5320, Dec. 2019, doi: 10.1109/TED.2019.2944647.

[13] V. I. Shcherbinin, A. V. Hlushchenko, A. V. Maksimenko, and V. I. Tkachenko, "Effect of cavity ohmic losses on efficiency of low-power terahertz gyrotron," IEEE Trans. Electron Devices, vol. 64, no. 9, pp 3898-3903, Sep. 2017, doi: 10.1109/TED.2017.2730252.

[14] V. I. Shcherbinin, T. I. Tkachova, and V. I. Tkachenko, "Improved cavity for broadband frequency-tunable gyrotron," IEEE Trans. Electron Devices, vol. 65, no. 1, pp. 257-262, Jan. 2018, doi: 10.1109/TED.2017.2769219.

[15] K. A. Avramides, I. G. Pagonakis, C. T. Iatrou, and J. L. Vomvoridis, "EURIDICE: A code-package for gyrotron interaction simulations and cavity design," in Proc. EPJ Web Conf., vol. 32, Sep. 2012, p. 04016, doi: $\underline{10.1051 / \text { epjconf } / 20123204016}$

[16] K. A. Avramidis, "Investigations and advanced concepts on gyrotron interaction modeling and simulations," Phys. Plasmas, vol. 22, no. 12, p. 123114, Aug. 2015, doi: 10.1063/1.4961962.

[17] V. L. Bratman, M. A Moiseev, M. I. Petelin, and R. É. Érm, "Theory of gyrotrons with a nonfixed structure of the high-frequency field," Radiophys. Quantum Electron., vol. 16, no. 4, pp. 474-480, Apr. 1973, doi: 10.1007/BF01030898

[18] V. A. Flyagin, A. V. Gaponov, I. Petelin, and V. K. Yulpatov, "The Gyrotron," IEEE Trans. Microw. Theory Techn., vol. 25, no. 6, pp. 514521, Jun. 1977, doi: 10.1109/TMTT.1977.1129149.

[19] J. J. Barroso, and R. A. Correa, "Design of a TE42,7 coaxial cavity for a $1 \mathrm{MW}, 280 \mathrm{GHz}$ gyrotron," Int. J. Infrared Millim. Waves, vol. 13, no. 4, pp. 443-455, Apr. 1992, doi: 10.1007/BF01010704.

[20] C. T. Iatrou, "Mode selective properties of coaxial gyrotron resonators," IEEE Trans. Plasma Sci., vol. 24, no. 3, pp. 596-605, Jun. 1996, doi: $10.1109 / 27.532942$

[21] Axon-cable.com (2016), "Thin wall PTFE tubings," [Online]. Available: http://www.axon-cable.com/publications/GAINEPTFEang.pdf. [Accessed: 31- Jan- 2020].

[22] V. E. Zapevalov, S. A. Malygin, V. G. Pavel'ev, and S. E. Tsimring, "Coupled-resonator gyrotrons with mode conversion," Radiophys. Quantum Electron., vol. 27, no. 9, pp. 846-852, Sep. 1984, doi: 10.1007/BF01041396.

[23] O. Dumbrajs, and E. Borie, "A complex cavity with mode conversion for gyrotrons,” Int. J. Electron., vol. 65, no. 3, pp. 285-295, Nov. 1988, doi: $10.1080 / 00207218808945230$.

[24] A. V. Maksimenko, V. I. Shcherbinin, A. V. Hlushchenko, V. I. Tkachenko, K. A. Avramidis and J. Jelonnek, "Starting currents for eigenmodes of a gyrotron cavity with mode conversion," IEEE Trans. Electron Devices, vol. 66, no. 3, pp. 1552-1558, Mar. 2019, doi: 10.1109/TED.2019.2893888.

[25] V. I. Shcherbinin, G. I. Zaginaylov, and V. I. Tkachenko, "HE and EH hybrid waves in a circular dielectric waveguide with an anisotropic impedance surface," Problems Atomic Sci. Technol., no. 4 (98), pp. 8993, Sep. 2015.

[26] V. I. Shcherbinin, G. I. Zaginaylov, and V. I. Tkachenko, "Cavity with distributed dielectric coating for subterahertz second-harmonic gyrotron," Problems Atomic Sci. Technol., no. 6 (106), pp. 255-258, Dec. 2016.

[27] V. I. Shcherbinin, G. I. Zaginaylov, and V. I. Tkachenko, "Analogy between circular core-cladding and impedance waveguides and their membrane functions," Prog. Electromagn. Res. M, vol. 53, pp. 111-120, 2017, 10.2528/PIERM16110902.

[28] V. I. Shcherbinin, and V. I. Tkachenko, "Cylindrical cavity with distributed longitudinal corrugations for second harmonic gyrotron," $J$. Infrared Millim. Terahertz Waves, vol. 38, no. 7, pp. 838-852, Jul. 2017, doi: 10.1007/s10762-017-0386-x.

[29] V. I. Shcherbinin, B. A. Kochetov, A. V. Hlushchenko, and V. I. Tkachenko, "Cutoff frequencies of a dielectric-loaded rectangular waveguide with arbitrary anisotropic surface impedance," IEEE Trans. Microw. Theory Techn., vol. 67, no. 2, pp. 577-583, Feb. 2019, doi: 10.1109/TMTT.2018.2882493.

[30] S. Antipov, C. Jing, A. Kanareykin, J. E. Butler, V. Yakimenko, M. Fedurin, K. Kusche, and W. Gai, "Experimental demonstration of wakefield effects in a THz planar diamond accelerating structure," Appl. Phys. Lett., vol. 100, no 13, p. 132910, Mar. 2012, doi: $10.1063 / 1.3697640$

[31] E. A. Nanni, W. R. Huang, K.-H. Hong, K. Ravi, A. Fallahi, G. Moriena, R. J. D. Miller, F. X. Kärtner, "Terahertz-driven linear electron acceleration," Nature Commun., vol. 6, no. 1, p. 8486, Dec. 2015, doi: $10.1038 /$ ncomms 9486 .

[32] B.D. O’Shea, G. Andonian, S. K. Barber, K. L. Fitzmorris, S. Hakimi, J. Harrison, P. D. Hoang, M. J. Hogan, B. Naranjo, O. B. Williams, V. Yakimenko, and J. B. Rosenzweig, "Observation of acceleration and deceleration in gigaelectron-volt-per-meter gradient dielectric wakefield accelerators," Nature Commun., vol. 7, p. 12763, Sep. 2016, doi: $\underline{10.1038 / \text { ncomms } 12763}$ 